\title{
Morphological Properties of Ultrahigh Molecular Weight Polyethylene and Low Molecular Weight Polypropylene Blend Gel Films
}

\author{
Tetsuya Ogita, Yoshihisa Kawahara, Chie Sawatari, ${ }^{*}$ \\ Fumihiko OzaKI, and Masaru Matsuo**, + \\ Department of Polymer Materials Engineering, Faculty of Engineering, \\ Yamagata University, Yonezawa 992, Japan \\ * Faculty of Eduction, Shizuoka University, Shizuoka 542, Japan \\ ** Department of Clothing Science, Faculty of Home Economics, \\ Nara Women's University, Nara 630, Japan
}

(Received August 23, 1990)

\begin{abstract}
Polyethylene-polypropylene blends were produced by gelation/crystallization from semidilute solutions by using ultrahigh molecular weight polyethylene (UHMWPE) $\left(3 \times 10^{6}\right)$ and low molecular weight polypropylene (LMWPP) $\left(2.2 \times 10^{5}\right)$. The polyethylene/polypropylene (PE/PP) compositions were $80 / 20,60 / 40,40 / 60,25 / 75$, and 20/80. The blend gels except the $20 / 80$ composition formed films. The maximum drawability was affected by composition. This phenomenon is discussed in terms of the morphology of blend films as studied by wide-angle X-ray diffraction, small-angle X-ray scattering, small-angle light scattering, optical microscopy, and birefringence. It turned out that the facile drawability of the blend films with draw ratio of 230 is due to the existence of a suitable level of entanglement meshes between UHMWPE and LMWPP chains in spite of their incompatibility in solution.
\end{abstract}

KEY WORDS Polyethylene-Polypropylene Blends / Polyethylene/Polypropylene (PE/PP) Compositions / Gelation/Crystallization / Maximum Drawability / Entanglement Meshes /

It is well known that the ultimate value of the Young's modulus is close to the crystal lattice modulus in the direction of the polymer chain axis. The crystal lattice moduli of polyethylene and polypropylene were measured by X-ray diffraction using ultradrawn films produced by gelation/crystallization from dilute solutions according to the method of Smith et al. ${ }^{1-4}$ According to previous works, the crystal lattice modulus of polyethylene, as measured by X-ray diffraction, was in the range $213-229 \mathrm{GPa}^{5}$ while that of polypropylene was $39-43$ GP. ${ }^{6}$ Furthermore, efforts have been made to produce polyethylene and polypropylene specimens whose Young's moduli are almost equal to their crystal lattice moduli. ${ }^{5-7}$ It was found that the Young's moduli of polyethylene and polypropylene approached $216^{5}$ and $40.4 \mathrm{GPa},{ }^{7}$ respectively, when the dry gel films of polyethylene and polypropylene could be consistently elongated to remarkably high draw ratios of 400 and 100 , respectively. The thermal properties of ultradrawn polyethylene and polypropylene films by differential scanning calorimetry (DSC) measurements provided the fact that the apparent melting point of polyethylene with draw ratio $(\lambda)$ in excess of 100 was $155^{\circ} \mathrm{C}$ higher than the equilibrium melting point $145.5^{\circ} \mathrm{C},{ }^{8}$ while the melting point of polypropylene with $\lambda=100$ was $178^{\circ} \mathrm{C}$ being close to the equilibrium melting point reported by Krigbaum and

\footnotetext{
† To whom all correspondence should be addressed.
} 
Uematsu. ${ }^{9}$ Accordingly, it is evident that the mechanical properties of ultradrawn polyethylene are much better than those of ultradrawn polypropylene but the thermal properties are poorer. Ultradrawing of ultrahigh molecular weight polyethylene-polypropylene blend film was thus carried out to improve high-impact strength and low-temperature toughness in a previous paper. ${ }^{10,11}$

In the previous paper, ${ }^{10}$ the morphological properties of the blend films, the polyethylene/ polypropylene (PE/PP) compositions being $75 / 25,50 / 50$, and $25 / 75$, were studied as a function of draw ratio. It was found that under scanning electron microscopy (SEM) and optical microscopy (cross-polarized) (POM) the textures within a drawn blend film are generally fibrillar with no indication of separate polyethylene and polypropylene domains, indicating intimate mixing of the two components despite their known incompatibility in solution. This phenomenon was also confirmed by wide-angle X-ray diffraction (WAXD) patterns during heating and cooling processes. The WAXD patterns revealed that within $25 / 75$ blend film drawn to $\lambda=60$, polyethylene crystallites within polypropylene matrix are evidently not free to allow random orientation in the melt state. This effect was thought to be due to the existence of a suitable level of entanglement meshes between polyethylene and polypropylene chains, which hampers the growth of separate phases of the two polymers in the gelation/crystallization process. ${ }^{10}$ The existence of entanglement meshes between polymer chains to assure high drawability was aslo confirmed using ultrahigh molecular weight polyethylene (UHMWPE) and low molecular weight polyethylene (LMWPE) blend films by WAXD patterns under heating process. ${ }^{11}$

In the present work, our focus is on drawing of UHMWPE-LMWPP blend films with different compositions produced by gelation/ crystallization from semidilute solution to study the possibility of the existence of en- tanglement meshes between UHMWPE and LMWPP chains in terms of drawability. This is of interest to study the relationship between incompatibility in solution and ability to form a gel film in relation to molecular chain length (molecular weight). The deformation mechanism of the resultant gel films with different compositions was investigated by small-angle X-ray scattering (SAXS), WAXD, small-angle light scattering (SALS), SEM, POM, and birefringence.

\section{EXPERIMENTALS}

The samples used in this experiment were linear polyethylene with average molecular weight $\bar{M}_{v}=3 \times 10^{6}$ (Mitsui Petrochemical Industry Ltd., Hizex Million 240M) and isotactic polypropylene with $\bar{M}_{v}=2.2 \times 10^{5}$ (Nihon Petrochemical Industry Ltd., J-130G). The PE/PP compositions were $80 / 20,60 / 40$, $40 / 60,25 / 75$, and $20 / 80$. The solvent was decalin. The concentration of polyethylenepolypropylene solution to assure maximum draw ratio of each blend film was determined through trial and error by the sample elongation in a hot-oven at $135^{\circ} \mathrm{C}$ under nitrogen flow. The results are listed in Table I. The solutions were stabilized with $0.1 \mathrm{w} / \mathrm{w} \%$ antioxidant, di- $t$-butyl- $p$-cresol. Decalin solutions were prepared by heating the well-blended polymer/solvent mixture at $135^{\circ} \mathrm{C}$ for $2 \mathrm{~h}$ under nitrogen. The hot homogenized solution was quenched by pouring it into an aluminum tray at room temperature, thus generating a gel. Decalin was allowed to evaporate from the gel under ambient conditions. The resultant dry gel, which had a thickness of $300 \mu \mathrm{m}$, was vacuum-dried for 1 day to remove residual traces of decalin.

The $25 / 75$ blend gel has ability to form a film while the $20 / 80$ blend gel has no such ability and is cracked on the drying. This means that an increase of the LMWPP content hampers to form a blend gel film and the 25/75 composition is a critical one as long as the given 
UHMWPE and LMWPP are used as specimens. This phenomenon is similar to the finding that LMWPE gels with $\bar{M}_{v}<3 \times 10^{5}$ have no ability to form films. ${ }^{12}$ Thus, the following experiments were carried out for the gel films with the $80 / 20,60 / 40,40 / 60$, and $25 / 75$ compositions.

The density, SAXS, WAXD, SALS, and DSC measurements were carried out to study morphological properties, which were described already elsewhere..$^{5-7}$

\section{RESULTS AND DISCUSSION}

The blend gel films could be stretched in a hot oven at $135^{\circ} \mathrm{C}$ under nitrogen. This is different from the elongation conditions in the case of UHMWPE-UHMWPP blend films. ${ }^{10}$ The UHMWPE-UHMWPP blend films could not be stretched in a hot oven at any temperature and then elongation was carried out in a poly(ethylene glycol) (PEG) bath. Successfully drawing in a hot oven at $135^{\circ} \mathrm{C}$ is due to the fact that the melting point of LMWPP is lower than that of UHMWPP. The maximum draw ratio depends on the $\mathrm{PE} / \mathrm{PP}$ compositions. This is shown in Table II. The drawability is less pronounced as the content of polypropylene increases. This suggests that the long chain of polyethylene plays an important role to form entanglement meshes with a short chain of polypropylene and slight decrease in UHMWPE content causes drastic decrease in the number of entanglement meshes to assure high drawability.

As summerized in Tables I and II, the maximum draw ratio is dependent upon the UHMWPE/LMWPP composition to form a blend gel. In order to study this reason, a series of experiments were carried out on the relationship between morphology and mechanical properties of the blend gel films.

Figure 1 shows the change in density as a function of draw ratio $\lambda$ for blend films with four PE/PP compositions and polyethylene homopolymer. The density increases with
Table I. Content of polyethylene and polypropylene at various $\mathrm{PE} / \mathrm{PP}$ compositions: $(\mathrm{g} / 100 \mathrm{ml})$

\begin{tabular}{clllll}
\hline $\begin{array}{c}\text { PE/PP } \\
\text { composition }\end{array}$ & $100 / 0$ & $80 / 20$ & $60 / 40$ & $40 / 60$ & $25 / 75$ \\
\hline PE & 0.65 & 0.64 & 0.62 & 0.58 & 0.52 \\
PP & 0 & 0.16 & 0.41 & 0.87 & 1.57 \\
\hline
\end{tabular}

Table II. Values of maximum draw ratios at various $\mathrm{PE} / \mathrm{PP}$ compositions

\begin{tabular}{cccccc}
\hline $\begin{array}{c}\text { PE/PP } \\
\text { composition }\end{array}$ & $100 / 0$ & $80 / 20$ & $60 / 40$ & $40 / 60$ & $25 / 75$ \\
\hline $\begin{array}{c}\text { Maximum } \\
\text { draw ratio }\end{array}$ & 400 & 200 & 50 & 40 & 30 \\
\hline
\end{tabular}

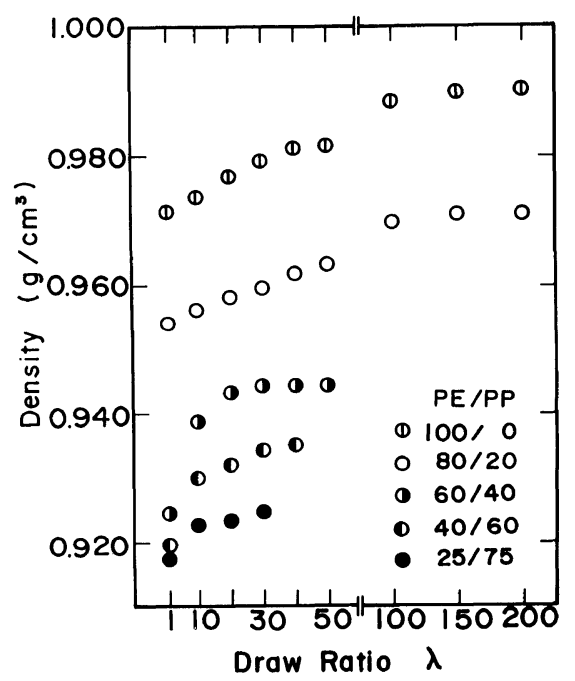

Figure 1. Changes in densities of the $80 / 20,60 / 40,40 / 60$, and $25 / 75$ blends and polyethylene homopolymer gel films against draw ratio $\lambda$.

increasing $\lambda$. The increase is related to the oriented crystallization.

Figure 2 shows WAXD and SAXS patterns (end view) from original (undrawn) blend films with the four PE/PP compositions and polyethylene homopolymer, when the incident beam was directed parallel to the film surface. The WAXD patterns exhibit preferential orientation of the $c$-axes of polyethylene and 

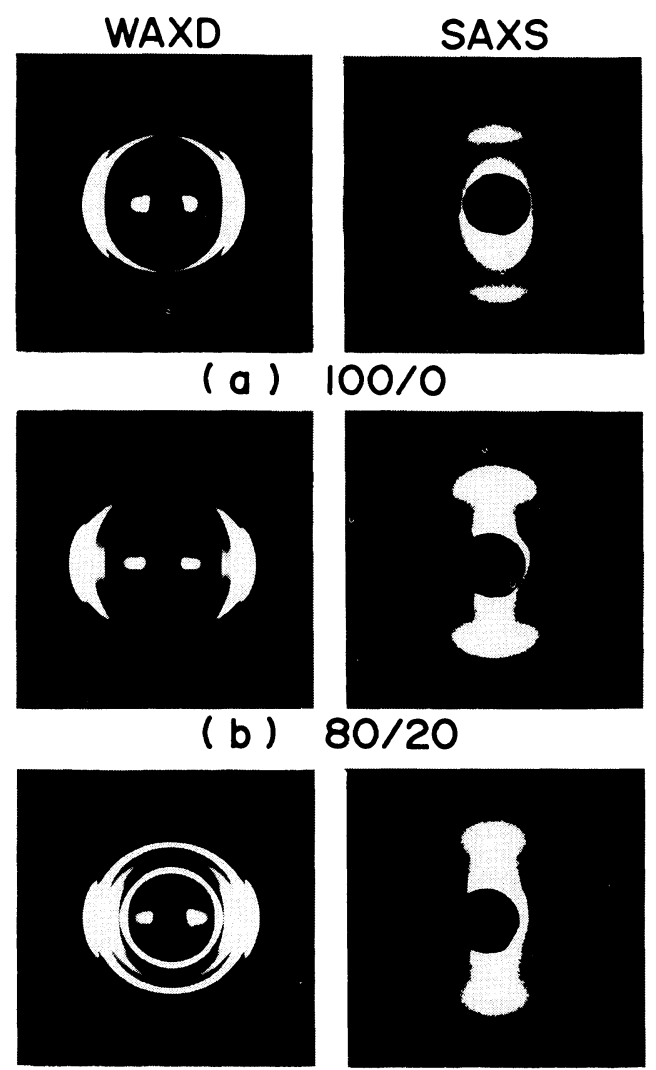

(c)
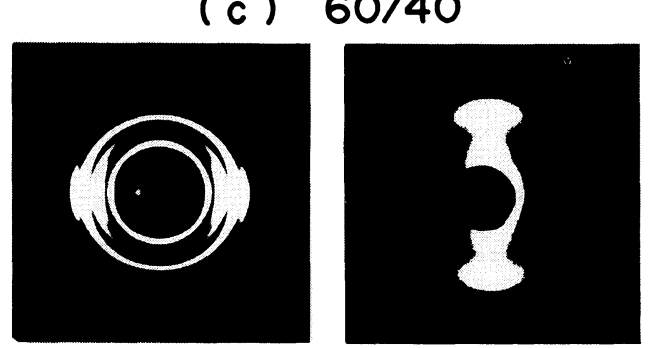

(d)

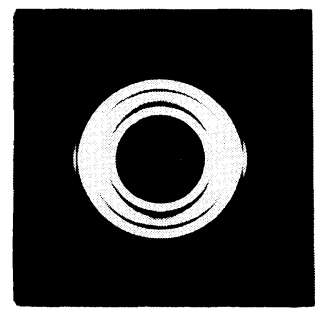

(e)

$40 / 60$

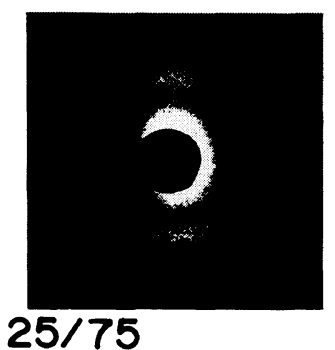

Figure 2. WAXD and SAXS patterns (end view) from the original blend gel films and the polyethylene gel film: (a) $100 / 0$, (b) $80 / 20$, (c) $60 / 40$, (d) $40 / 60$, and (e) $25 / 75$. polypropylene perpendicular to the film surface but the orientational degree is less pronounced with increasing the LMWPP content. The SAXS patterns show scattering maximum corresponding to a long period of about $115 \AA$ in the meridional direction. Considering the profiles of the SAXS patterns, it is obvious that the original (undrawn) blend films are composed of crystal lamellae that are more or less oriented with their large flat faces parallel to the film surface but the orientational fluctuation of the lamellae and the fluctuation in lamellar size increases with increasing the content of LWMPP. This is probably due to the fact that the existence of LMWPP hampers the formation of large lamellae of UHMWPE that have intrinsically small fluctuation in size and become highly oriented parallel to the film surface.

Figure 3 shows SEM, POM, and the corresponding $H_{\mathrm{v}}$ SALS patterns. At this scale of magnification, it was confirmed that the LMWPP chain within the blend gel film cannot form spherulites but rod-like textures, although the LMWPP melt film used in this experiment had spherulites. The corresponding SALS patterns from the four kinds of blend films display diffuse $\mathrm{X}$-type lobes. The intensity had a maximum in the center and decreased monotonically with increasing scattering angle. These observations are indicative of scattering from rod-like textures, the optical axes being oriented parallel or perpendicular to the rod axis. ${ }^{12}$ This supports observation under microscope.

The existence of rod-like textures has been observed for UHMWPE gel films prepared without stress. ${ }^{13,14}$ In contrast, the morphology of LMWPE gels (not film) showed spherulitic textures. ${ }^{15}$ Furthermore, it was confirmed that the patterns of UHMWPELMWPE blend gel film with the 50/50 composition are due to scattering both from rod-like of UHMWPE and from spherulitic textures of LMWPE. ${ }^{11}$ Accordingly, the existence of the rod-like texture of LMWPP 

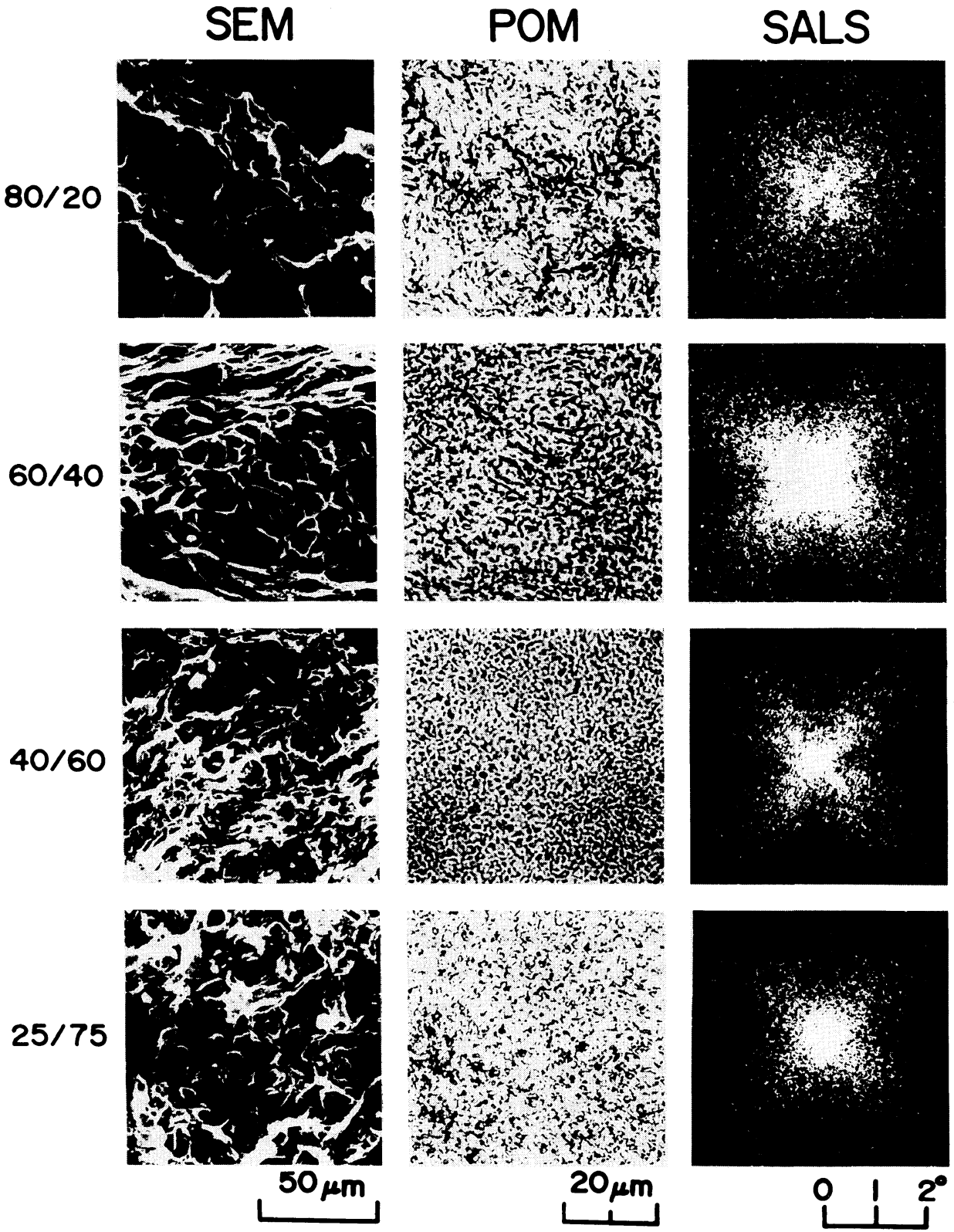

Figure 3. Scanning electron micrographs, polarized optical micrographs (cross polarized), and $H_{\mathrm{v}}$ light scattering patterns from the our kinds of original blend films.

within the PE/PP blend gel films prepared without stress, as in the case of this experiment, is of interest in contrast with the morphology of LMWPE within the UHMWPE-LMWPE blend gel films. It should be noted that the scatterning patterns appear at lower scattering angles and the notch of the four lobes is less clearly defined in comparison with the patterns observed for the HUMWPE-LMWPE blend gel films. ${ }^{16}$ Judging from the WAXD and 


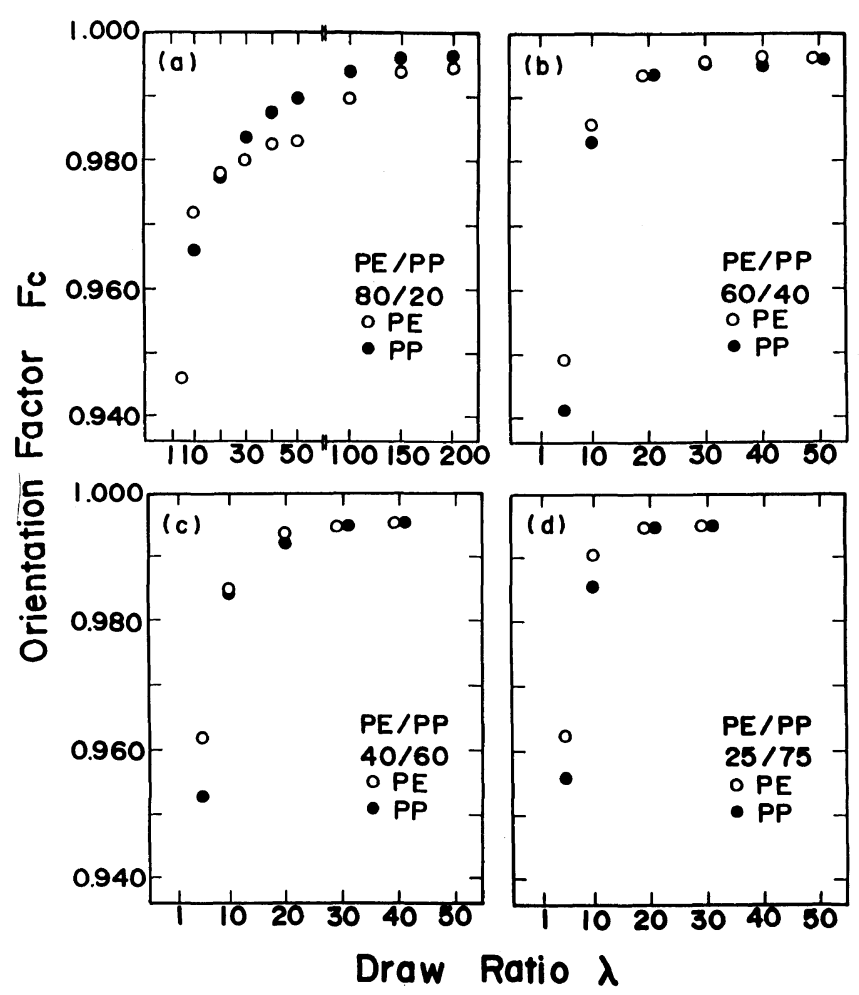

Figure 4. Second order orientation factors of the $c$-axes of polyethylene and polypropylene as a function of draw ratio within the four kinds of blend films.

SAXS patterns in Figure 2, such patterns presumably indicate the existence of large-size rods with finite length and width, the optical axes being oriented normal to the film surface but having orientational disorder with respect to the rod axis.

Figure 4 shows the second order orientation factors of the $c$-axes of polyethylene and polypropylene measured for four kinds of blend films, respectively. The orientation factors of the polyethylene were estimated directly from the orientation distribution function of the $\left(\begin{array}{lll}0 & 0 & 2\end{array}\right)$ plane and that of polypropylene was derived from the orientation factors of the (lll 110$),\left(\begin{array}{lll}0 & 4 & 0\end{array}\right)$, and ( $\left.\begin{array}{lll}1 & 3 & 0\end{array}\right)$ planes using the method of Roe and Krigbaum. ${ }^{17}$ The peak separation of the blend system was described in detail elsewhere. ${ }^{11}$ As can be seen in these figures, a high orientational degree of polyethylene and polypropylene crystallites can be realized at $\lambda=10$ and with further elongation the $c$-axes tend to orient almost parallel to the stretching direction. The preferential orientation of the $c$-axes of polyethylene is less pronounced in comparison with that of polypropylene for the $80 / 20$ blend film with draw ratios beyond $\lambda=20$ but for the other blend films the orientational degrees of polyethylene are slightly higher than and/or almost equal to those of polypropylene.

In order to assess the UHMWPE/LMWPP composition-dependence of the orientation factor, the oreintation factors were plotted against the UHMWPE/LMWPP composition, based on the results in Figure 4. Figure 5 shows the results. With increasing LMWPP content, both orientation factors of polyethylene and polypropylene become higher. Such behavior is quite different from the molecular orientation of polyethylene within 

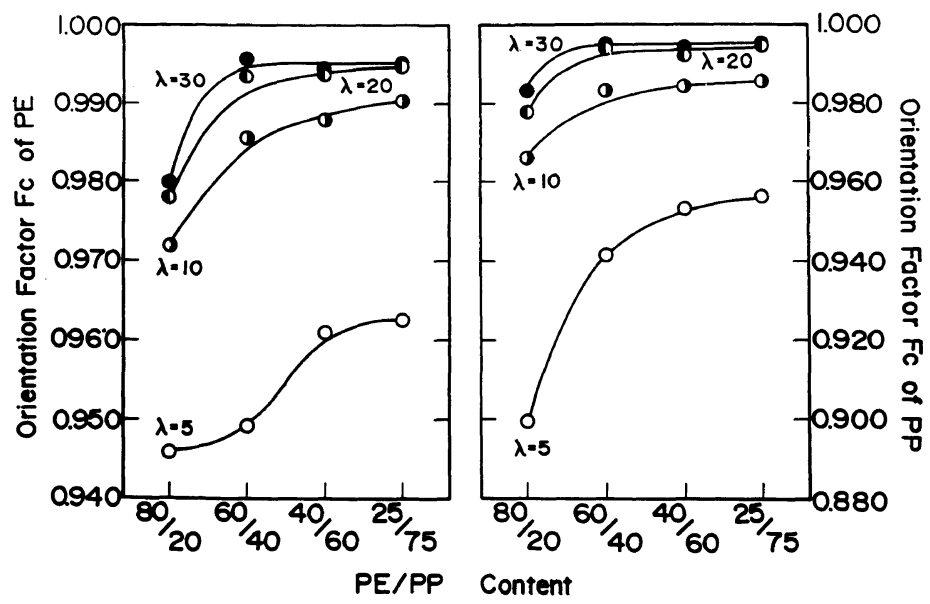

Figure 5. Second order orientation factors of the $c$-axes of polyethylene and polypropylene within the blend gel films with the indicated PE/PP compositions at various draw ratios.

UHMWPE-UHMWPP blend films observed already in previous work. ${ }^{11}$ According to the previous paper, it was found that as for the blend film with $\lambda=20$, the orientation factor of the $c$-axis of polyethylene becomes lower with increasing polyethylene content as in the case of Figure 5 but reversely, the factor becomes higher with $\lambda \geqq 40$.

Figure 6 shows the Young's modulus as a function of draw ratio $\lambda$ for the blend films with four UHMWPE/LMWPP compositions. The values increase with increasing $\lambda$. This tendency is more pronounced with increasing polyethylene content, although the orientation factor of the $c$-axes of polyethylene is more pronounced as LMWPP content increases as shown in Figure 5. This is probably attributed to the fact that the crystal lattice modulus of polyethylene is much higher than that of polypropylene and the increase in orientation factors of polyethylene $c$-axes with increasing LMWPP content shown in Figure 5 is not significant enough to change the UHMWPE/ LMWPP compositions for the specimens drawn beyond $\lambda=10$.

Figure 7 shows tensile strength as a function of draw ratio $\lambda$ for the blend films. The value increases with increasing $\lambda$ and PE content for specimens with the same draw ratio. As shown

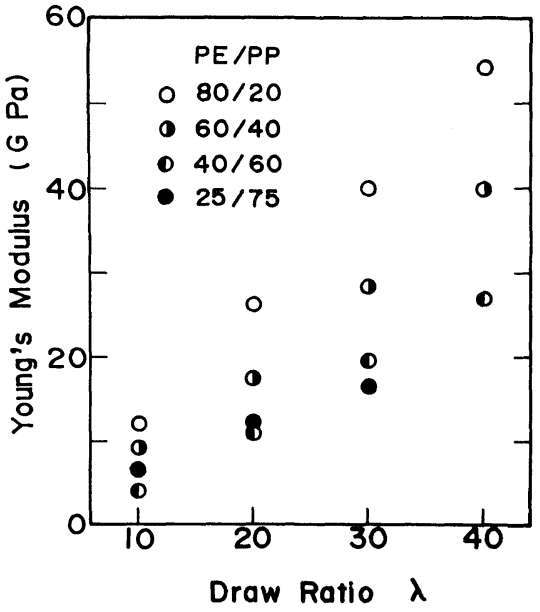

Figure 6. Young's modulus as a function of draw ratio of the four kinds of blend films.

in Figure 5, the orientation factor of the $c$ axes of polyethylene is hardly affected by the UHMWPE/LMWPP composition at beyond $\lambda=10$. Hence it is evident that the increase in tensile strength with draw ratio is attributed to the difference of intrinsic tensile strength between polyethylene and polypropylene.

Figures 8-11 show micrographs of POM and SALS patterns under $H_{\mathrm{v}}$ polarization condition as a function of draw ratio, observed for the $80 / 20,60 / 40,40 / 60$, and $25 / 75$ blend 


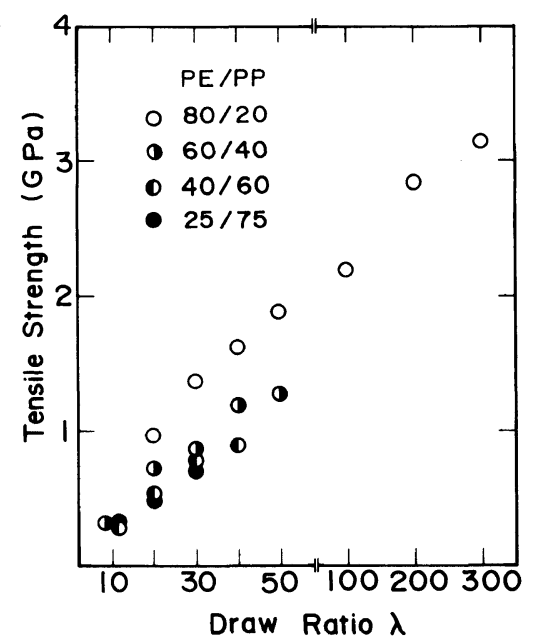

Figure 7. Tensile strength as a function of draw ratio of the four kinds of blend films.

films. The change of the patterns with draw ratio is sensitive to the compositions. In Figure 8 , for the $80 / 20$ composition, the SALS patterns at $\lambda=3$ and 5 show indistinct lobes in the horizontal direction and the corresponding micrographs exhibit fibrils oriented parallel to the stretching direction. Such indistinct lobes indicate disruption of rods by elongation due to unfolding of crystal lamellae, since the scattering pattern becomes larger than that in Figure 3(a). In the range of draw ratios from 5 to 20 times, drastic change of SALS patterns occurred but no change could observed in the corresponding micrographs. The sharp streaks in the patterns (c) and (d) correspond to the scattering from highly oriented fibrous textures observed in the corresponding micrographs. The X-type lobes imply scattering from oriented rods within the fibrous structure but the existence of the rods could not be confirmed in the micrographs. At $\lambda=30$, the SALS pattern shows a dark field of vision indicating the disappearance of rod-like texture due to rupture of rod-like texture.

Figure 9 shows the micrographs of POM and SALS patterns under the $H_{\mathrm{v}}$ polarization condition as a function of draw ratio $\lambda$, observed for the $60 / 40$ blend films. The SALS
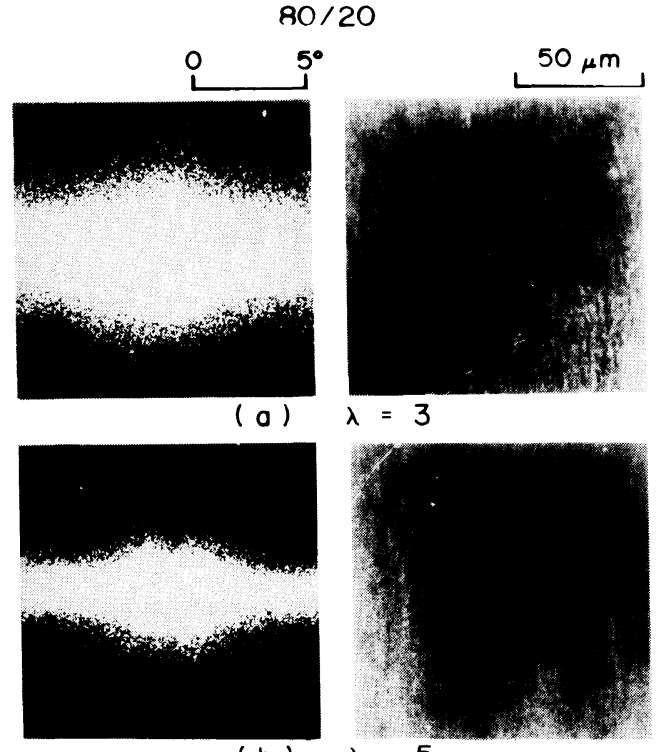

(b)
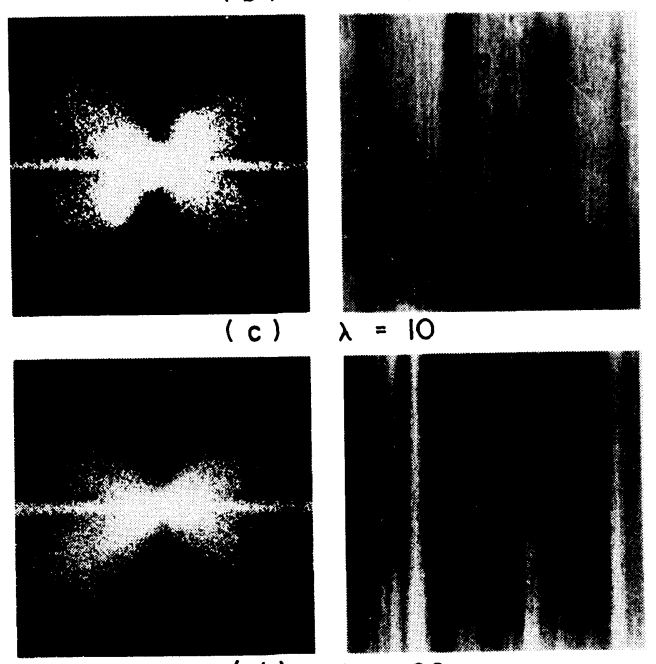

(d)

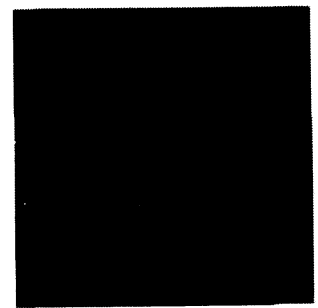

$\lambda=20$

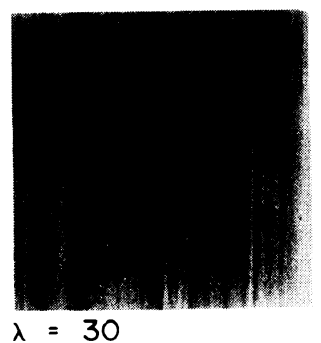

Figure 8. $H_{v}$ light scattering patterns and optical micrographs (cross polarized) for the $80 / 20$ blend films at various draw ratios $\lambda$. 


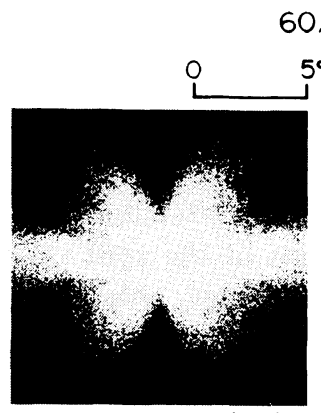

(a)

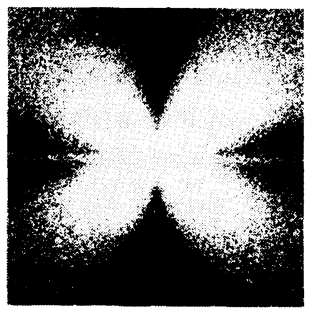

(b)

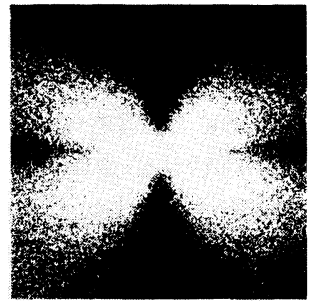

(c)

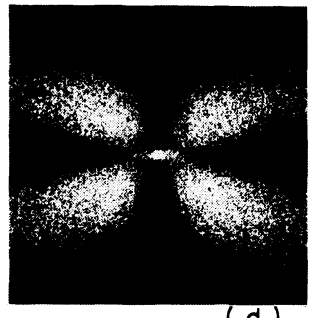

(d)

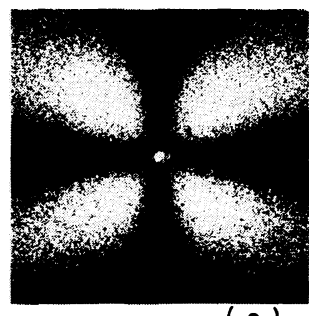

(e)

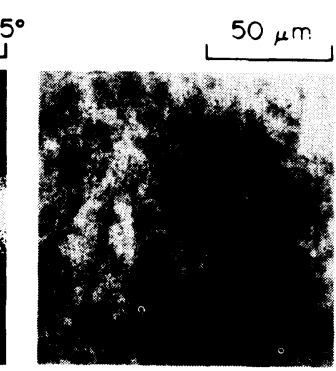

$\lambda=5$

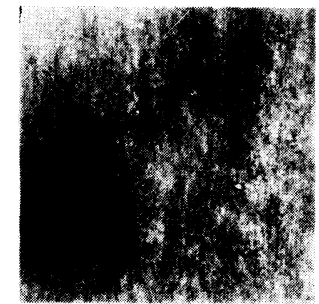

$\lambda=10$

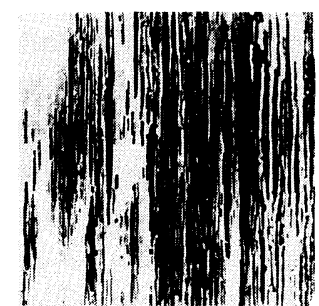

$\lambda=30$

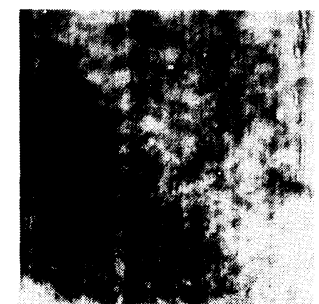

$\lambda=40$

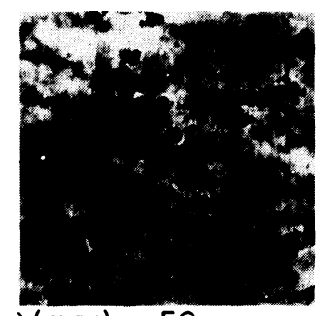

$\lambda(\max )=50$

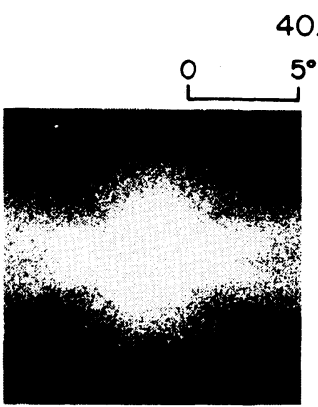

(a)

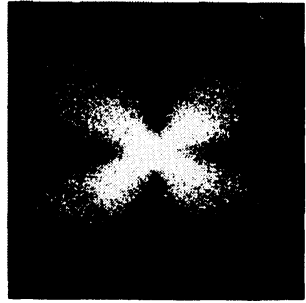

(b)

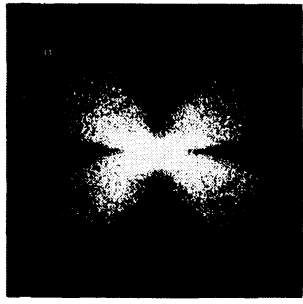

(c)

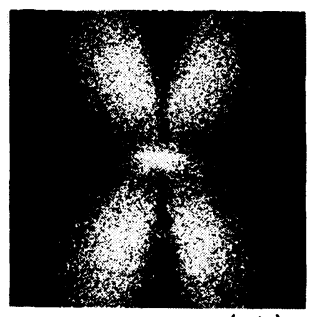

(d)

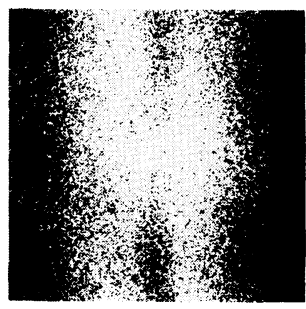

( e )
$0 / 60$

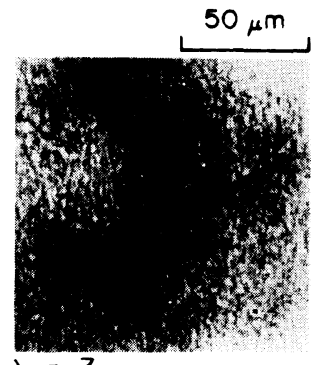

$\lambda=3$

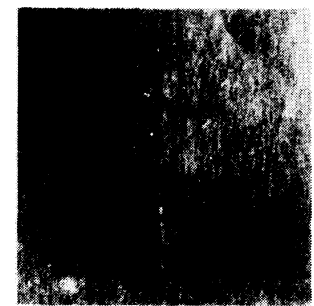

$\lambda=5$

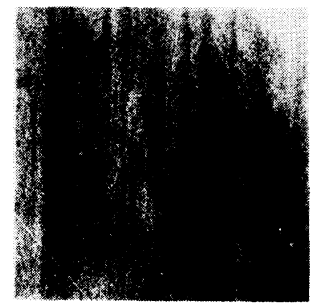

$\lambda=20$

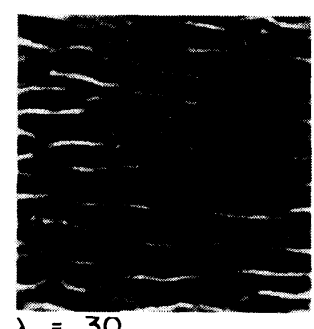

$\lambda=30$

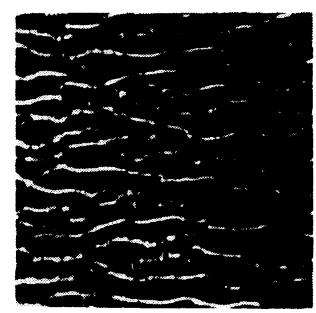

$\lambda(\max )=40$

Figure 9. $H_{\mathrm{v}}$ light scattering patterns and optical micrographs (cross polarized) for the $60 / 40$ blend films at various draw ratios $\lambda$.

Figure 10. $H_{\mathrm{v}}$ light scattering patterns and optical micrographs (cross polarized) for the $40 / 60$ blend films at various draw ratios $\lambda$. 


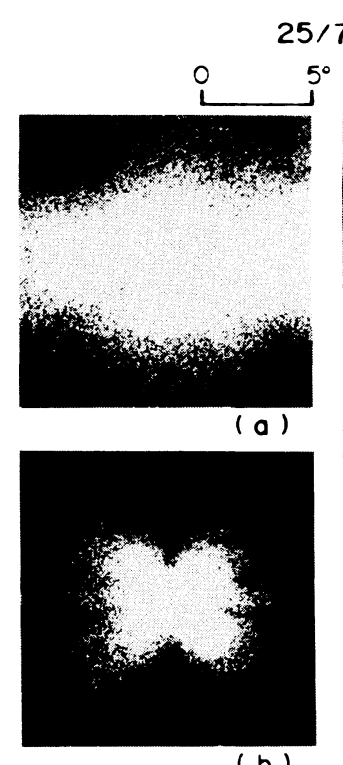

$25 / 75$

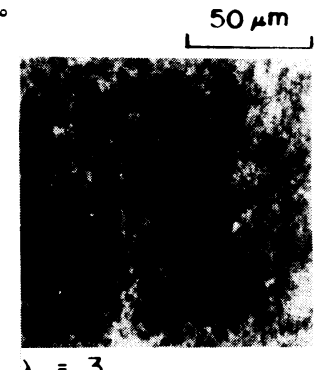

$\lambda=3$

( b )

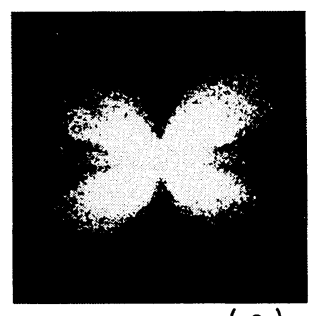

(c)

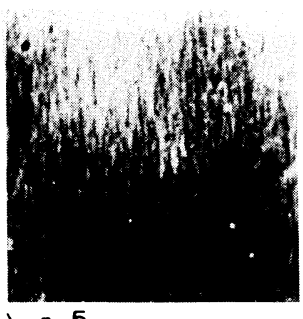

$\lambda=5$
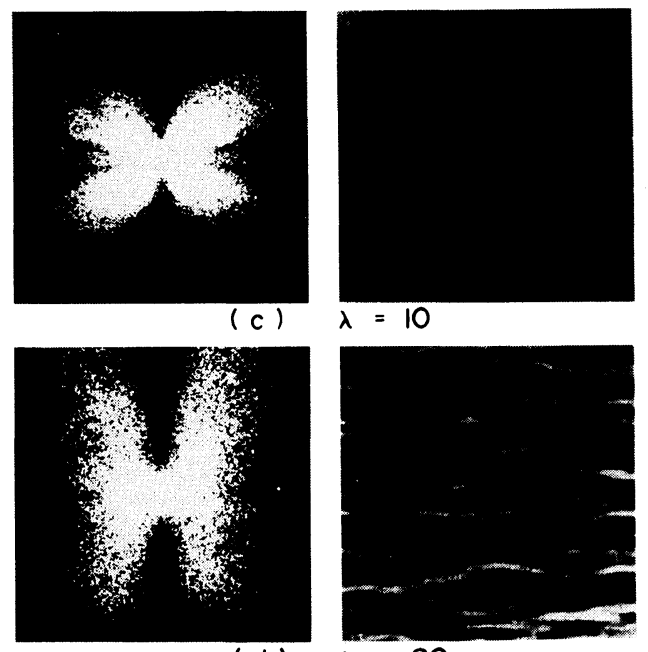

(d)

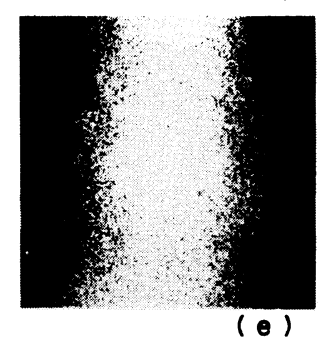

$\lambda=10$

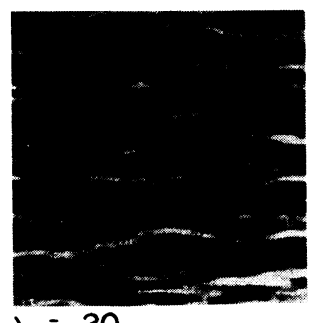

$\lambda=20$

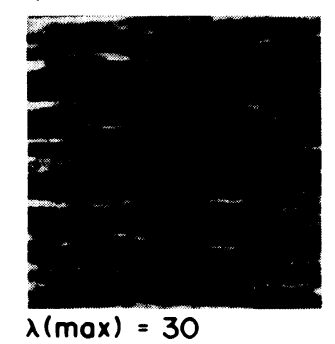

Figure 11. $H_{v}$ light scattering patterns and optical micrographs (cross polarized) for the $25 / 75$ blend films at various draw ratios $\lambda$. pattern at $\lambda=5$ shows the lobes extended in the meridional direction and broad streaks in the horizontal one. According to the theoretical analysis of SALS pattern, ${ }^{18}$ it may be postulated that the rod-like textures are oriented perpendicular to the stretching direction and the arrangement of the rods causes interference interparticle effects, although the morphology to give the change of SALS patterns cannot be discerned in the corresponding micrographs. Elongation up to $\lambda=$ 10 causes development of the X-type pattern with clear lobes at odd multiples of $45^{\circ}$. Such a profile indicates the random orientation of rods, the optical axes being oriented at 0 or $90^{\circ}$ with respect to the rod axis. ${ }^{12}$ As shown in Figure 4, however, the crystallites of polyethylene and polypropylene take preferential orientation with respect to the stretching direction. This indicates that the rods orient $\pm 45^{\circ}$ with respect to the stretching direction and the $c$-axes corresponding to the optical axes orient predominantly in the stretching direction. Such orientation can be distinguished easily, since the notch of the four lobes is more clearly defined than those observed for undrawn films generally as shown in Figure 3. With increase up to $\lambda=50$, the pattern shows the four leaf clover-type as has been observed for drawn film having spherulitic textures. The appearance of such a pattern is out of common sense of polymer science. In the corresponding micrograph, spherulitic structure cannot be confirmed. The appearance of spherulitic textures remains an unreasonable problem.

Figure 10 shows micrographs and SALS patterns for the blend films with the $40 / 60$ composition. The pattern at $\lambda=3$ shows indistinct scattering lobes extending in the horizontal direction. This also suggests arrangement of rods in the disruptive process of fibrillar texture as discussed before. The elongation up to $\lambda=5$ causes development of $\mathrm{X}$-type patterns whose lobes are slightly extended in the horizontal direction. As 
Morphological Properties of UHMWPE-LMWPP Blend Gel Films
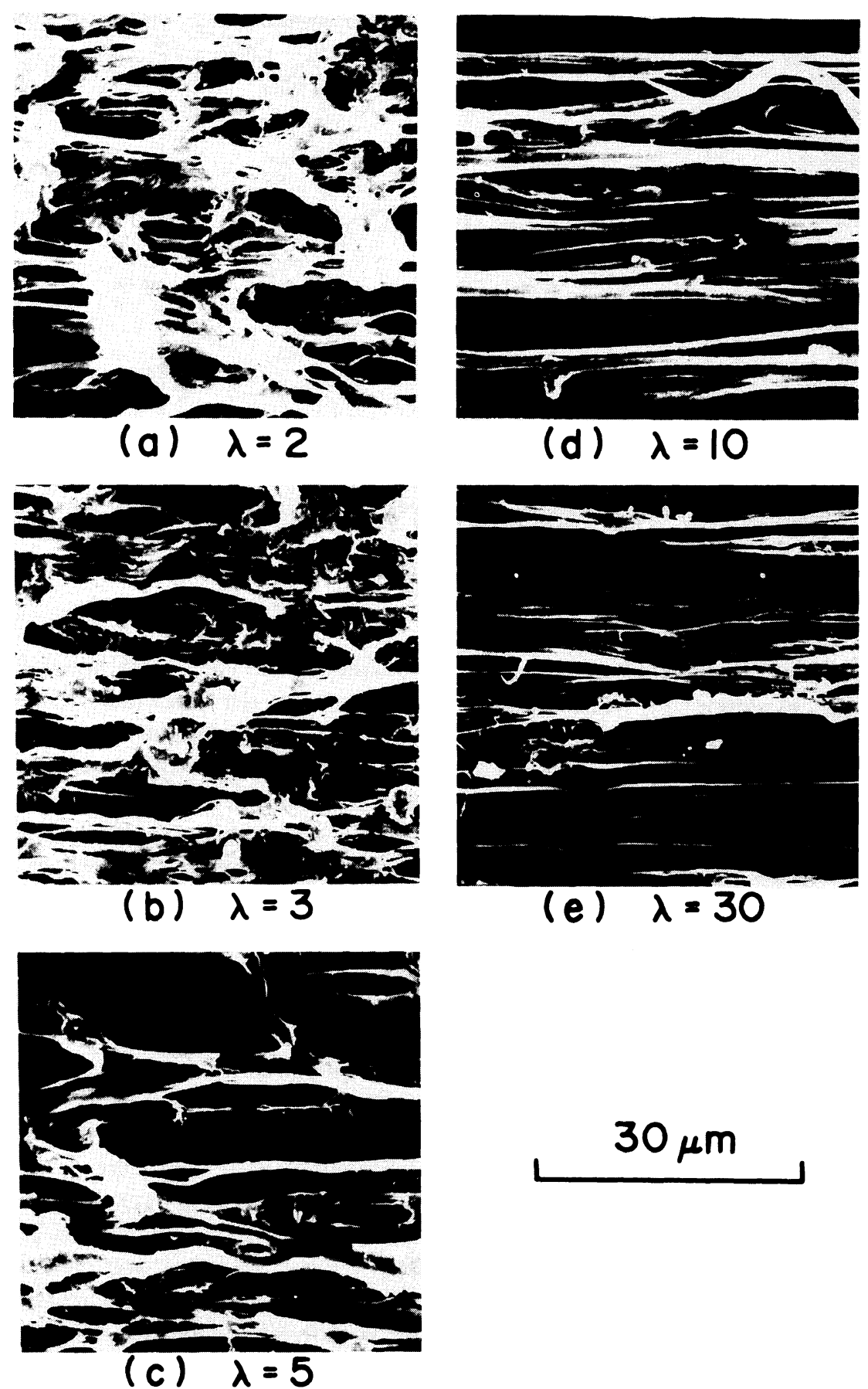

\section{$30 \mu \mathrm{m}$}

Figrue 12. Scanning electron micrographs of the $25 / 75$ blend films at various draw ratios $\lambda$. The stretching direction is horizontal one. 

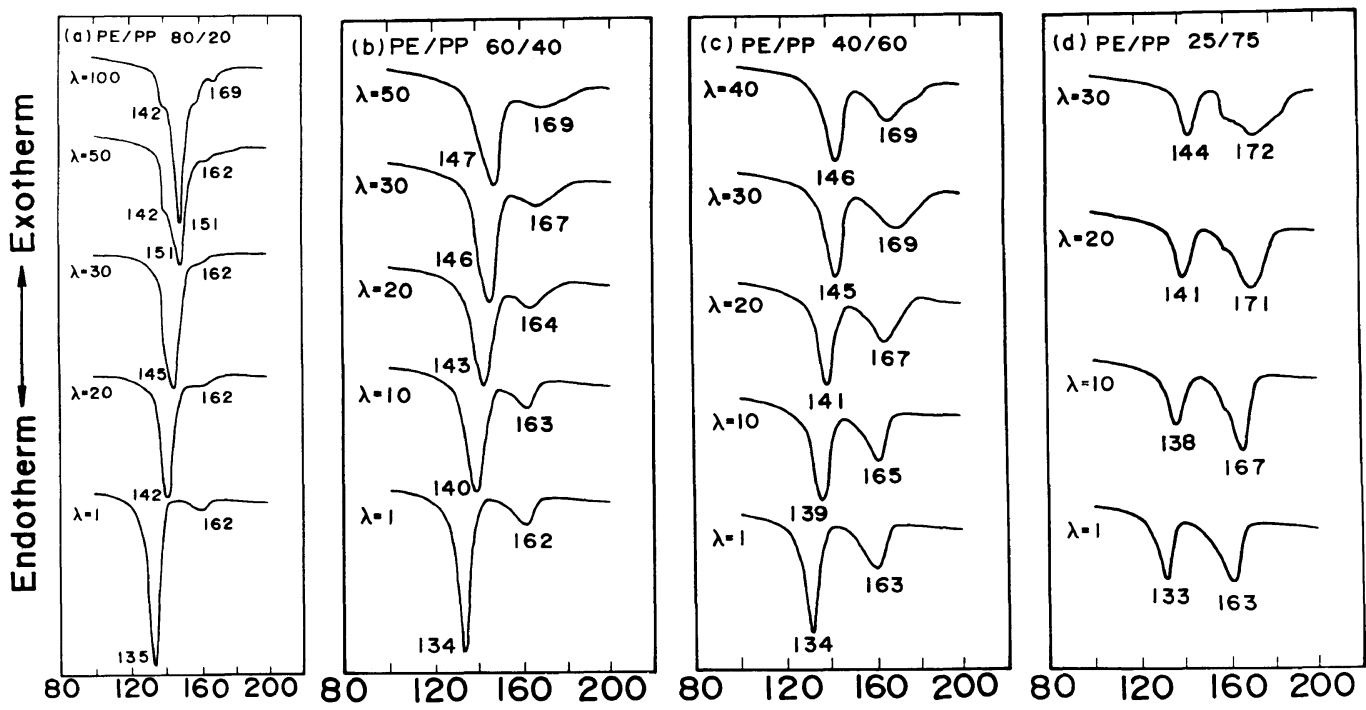

Figure 13. DSC curves of the four kinds of blend films drawn to various draw ratios $\lambda$ : (a) $80 / 20$, (b) $60 / 40$, (c) $40 / 60$, and (d) $25 / 75$.

discussed before, such a X-type pattern suggests the existence of rods oriented predominantly with respect to the stretching direction. At $\lambda=30$, the scattering lobes are extended in the meridional direction, indicating oriented crystallization and the corresponding micrograph reveals the appearance of large fibrils perpendicular to the stretching direction. Such a micrograph has never been observed for ultradrawn polyethylene, ${ }^{19}$ polypropylene, ${ }^{7}$ and their blend gel films. ${ }^{10}$ This information of the new structure, still remains an unresolved problem.

Figure 11 shows micrographs and SALS patterns for the blend film with the $25 / 75$ composition. The SALS pattern and micrograph at each draw ratio are similar to the profiles observed for the $40 / 60$ blend films shown in Figure 10.

Figure 12 shows the change in the appearance of $25 / 75$ blend films with draw ratio under SEM. As illustrated in the micrographs and in Figure 3 observed for an undrawn film, the fibrillar texture like spongy tissue comprised of interconnected lamellar crystal and with elongation, the cross-linking loci appeared to consist of fibrillar shish-kabab-like crystals arranged in the stretching direction. Such profiles can be observed for all blend films used in this experiment and the deformation mechanism of fibrillar texture of the blend films is also similar to that of polyethylene homopolymer reported elsewhere. ${ }^{19}$ The micrographs give detailed information of the fibrillar texture in comparison with those of POM. However, it is very difficult to understand the origin of the novel micrograph of POM shown in Figure 11(e) from the corresponding micrograph (e) in Figure 12.

Figure 13 shows the change in the profile of the DSC curves at a constant rate of $10^{\circ} \mathrm{C} \mathrm{min}^{-1}$ with increasing draw ratio $\lambda$, in which columns (a), (b), (c), and (d) correspond to the $80 / 20,60 / 40,40 / 60$, and $25 / 75$ blends, respectively. To check the reproducibility of the profiles, DSC measurements for a given draw ratio were carried out several times. As illustrated in Figure 13, the DSC curves exhibit separated endotherms corresponding to the individual homopolymers. The peak at the lower temperature is due to polyethylene and the other is due to polypropylene. Individual 
peaks shift to higher temperature with increasing draw ratio $\lambda$. At the same draw ratio, the melting point of polyethylene within the blends tends to be lower with increasing LMWPP content. This is probably due to the fact that crystal size of polyethylene within the blend films is smaller than that within the polyethylene homopolymer film.

Figure 14 shows the change in birefringence with increasing temperatures, observed for the $40 / 60$ and $25 / 75$ blend films with $\lambda=20$ and 30. The birefringence data is almost independent of temperature up to $170^{\circ} \mathrm{C}$. The value

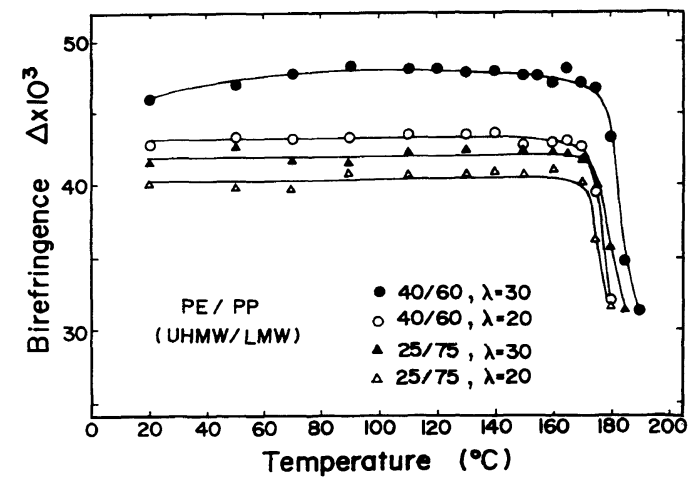

Figure 14. Change in birefringence as a function of temperature for the two kinds of the blend films with $\lambda=20$ and 30 . decreased drastically with further increase in temperature because of melting of polyethylene crystals but the specimens could be maintained. This supports that the blend films have a suitable number of entanglement meshes between UHMWPE and LMWPP chains enough to assure elongation.

In order to support this concept, WAXD patterns were observed during heating and cooling processes. Figure 15 shows the results for the $40 / 60$ blend films with $\lambda=20$ and 30 when the specimens were fixed at a constant stress of $0.015 \mathrm{GPa}$ to avoid shrinkage of the specimens. The specimens were annealed for $20 \mathrm{~min}$ at the indicated temperatures prior to photographing. As can be seen in a series of patterns, the diffraction intensities from the (1 10$)$ and (200) planes of polyethylene become weaker as temperature increases to $150^{\circ} \mathrm{C}$. The reflections from both the specimens disappeared due to the melting at 160 and $170^{\circ} \mathrm{C}$, respectively, but the specimens could be maintained. This is consistent with the birefringence data in Fig. 14. In the cooling process, it is seen that polyethylene crystllites within the polypropylene matrix are evidently not free to allow random orientation in the melt state.

\section{UHMWPE/LMWPP $\quad 40 / 60$}

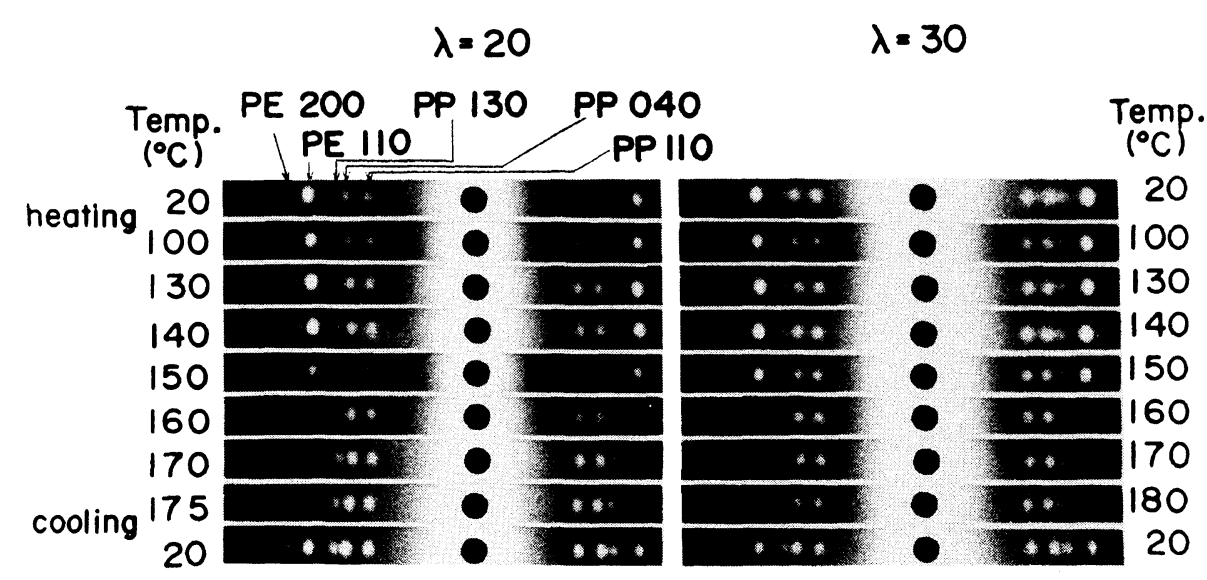

Figure 15. Change in WAXD patterns (through view) in the horizontal direction from the $40 / 60$ blend films $(\lambda=20$ and 30$)$ at the indicated temperatures during heating and cooling processes. 


\section{CONCLUSION}

Polyethylene-polypropylene blend films were prepared by gelation/crystallization from semidilute solutions by using ultrahigh molecular weight polyethylene $\left(\bar{M}_{v}=3 \times 10^{6}\right)$ and low molecular weight polypropylene $\left(\bar{M}_{v}=2.2 \times\right.$ $\left.10^{5}\right)$. The UHMWPE/LMWPP compositions were $80 / 20,60 / 40,40 / 60,25 / 75$, and $20 / 80$. The $20 / 80$ blend gel could not form a film but the other gels have ability to form a gel. The blend films could be readily elongated in a hot oven at $135^{\circ} \mathrm{C}$ under nitrogen. The morphological properties of the blend films were dependent upon the compositions. The texture is generally fibrillar with no indication of separate polyethylene and polypropylene domains under scanning electron microscopy and optical microscopy (cross-polarized). This - indicates intimate mixing of the two compositions despite their known incompatibility in solution. This effect is thought to be due to the existence of a suitable level of entanglements between UHMWPE and LMWPP chains. The drawability decreased with increasing LMWPP content, indicating the decrease in the number of entanglements due to wriggle of chains during elongation.

\section{REFERENCES}

1. P. Smith, P. J. Lemstra, B. Kalb, and A. J. Pennings,
Polym. Bull., 1, 733 (1979).

2. P. Smith and P. J. Lemstra, J. Mater. Sci., 15, 505 (1980).

3. P. Smith, P. J. Lemstra, and H. C. Booij, J. Polym. Sci., Polym. Phys. Ed., 19, 877 (1981).

4. P. Smith, P. J. Lemstra, J. P. L. Pijppers, and A. M. Kiel, Colloid Polym. Sci., 258, 1070 (1981).

5. M. Matsuo and C. Sawatari, Macromolecules, 19, 2036 (1986).

6. C. Sawatari and M. Matsuo, Macromolecules, 19, 2653 (1986).

7. M. Matsuo, C. Sawatari, and T. Nakano, Polym. J., 18, 759 (1986).

8. P. J. Flory and A. J. Vrij, J. Am. Chem. Soc., 85, 3548 (1963).

9. W. R. Krigbaum and I. Uematsu, J. Polym. Sci., Polym. Phys. Ed., A-2, 3, 767 (1965).

10. C. Sawatari, S. Shimogiri, and M. Matsuo, Macromolecules, 20, 1033 (1987).

11. C. Sawatari, S. Satoh, and M. Matsuo, Polymer, 31, 1456 (1990).

12. M. B. Rhodes and R. S. Stein, J. Polym. Sci., A-2, 7, 1538 (1969).

13. M. Matsuo and R. St. J. Manley, Macromolecules, 15, 985 (1982).

14. M. Matsuo and R. St. J. Manley, Macromolecules, 16, 1500 (1983)

15. C. Sawatari, T. Okamura, and M. Matsuo, Polym. J., 18, 741 (1986).

16. C. Sawatari and M. Matsuo, Polymer, 30, 1603 (1990).

17. R. J. Roe and W. R. Krigbaum, J. Chem. Phys., 40, 2608 (1964).

18. C. Sawatari, M. Iida, and M. Matsuo, Macromolecules, 17, 1765 (1984).

19. M. Matsuo, K. Inoue, and N. Abumiya, Sen- $i$ Gakkaishi, 36, 696 (1984). 